\title{
Electrosurgery and clinical applications of electrosurgical devices in gynecologic procedures
}

\author{
Soheila Aminimoghaddam ${ }^{1}$, Rozhin Pahlevani ${ }^{2 *}$, Maryam Kazemi ${ }^{2}$ \\ Received: 12 Sep 2017 \\ Published: 22 Sep 2018
}

\begin{abstract}
Background: Electrosurgery is widely used in reproductive related surgeries and technological advancements to improve efficacy and reduce potential complications. However, some reports have indicated lack of sufficient knowledge and training about basic principles and technical aspects of electrosurgery among obstetricians and gynecologists.

Methods: In this paper we present a summary on basic concepts and principles of electrosurgery and review the recent evidence on the use of electrosurgical devices in gynecologic procedures including endometrial ablation, gynecologic malignancies, loop electrode excision procedure (LEEP), and infertility.

Result: Considering the extensive use of these technologies in reproductive related surgeries, procedures including laparoscopy, hysteroscopy, and loop procedures further highlights the importance of more detailed training in this field. Gynecologists must learn the basics in more detail and update their knowledge on the growing body of evidence regarding the advancements of these technologies to reduce potential complications and select the most cost-effective treatment options for each patient.

Conclusion: Try to understanding the underlying biophysical principles and more in-depth familiarity with various electrosurgical devices could lead to less complications and optimize evidence-based gynecological practice.
\end{abstract}

Keywords: Electrosurgery, Gynecologic procedures

Copyright $\odot$ Iran University of Medical Sciences

Cite this article as: Aminimoghaddam S, Pahlevani R, Kazemi M. A brief overview on the basic concepts of electrosurgery and clinical applications of electrosurgical devices in gynecologic procedures. Med J Islam Repub Iran. 2018 (22 Sep);32:90. https://doi.org/10.14196/mjiri.32.90

\section{Introduction}

Electrosurgery is defined as the use of high frequency electric current to desiccate, coagulate, and fulgurate biological tissues (1). Using heat to stop bleeding or achieve other medical purposes has a long history, and the earliest documents referring to it dates back to Albucasis in 980 BC who described the use of hot iron in a concept similar to electrocautery to control bleeding in patients (2). In the 19th century, Arsened'Arsonval, a French physician, reported that frequencies above $10 \mathrm{kHz}$ only lead to heat generation in biological tissues without causing neuromuscular stimulation. Later, Oudin described the successful use of a device designed based on this concept to destroy biological tissues. In the 20th century, the collaboration of a leading neurosurgeon Harvey Cushing and a Harvard physicist William Bovie resulted in development of modern electrosurgical equipment capable of cutting, desiccation, and coagulation.

Corresponding author:Dr Rozhin Pahlevani, rozhin_p92@yahoo.com

1. Department of Gynecology, Firoozgar Hospital, Iran University of Medical Sciences, Tehran, Iran

2. School of Medicine, International Campus (IC), Iran Medical University of Medical Sciences, Tehran, Iran
Since then, many technological advancements and various modification of designs have been introduced and widely used in different surgical fields to reduce potential complications (3).

Due to their extensive use in many surgical procedures and the clinical and cost-effectiveness of electrosurgical devices and techniques, clinicians should be familiar with their basic principles. Consequently, surgeons must gain enough expertise about the potential complications of electrosurgery and make sense of the possible technical faults of the commonly used instruments. The wide use of electrosurgery in gynecological procedures including laparoscopy, hysteroscopy, and loop procedures further highlights the importance of more detailed training in this field. However, there remains a knowledge gap and lack of systematic training in the field of reproductive related surgery.

$\uparrow$ What is "already known" in this topic:

Electrosurgery is widely used in reproductive related surgeries, and technological advancements to improve efficacy and reduce potential complications are rapidly advancing minimally invasive gynecological procedures.

$\rightarrow$ What this article adds:

We reviewed the recent evidence on the use of electrosurgical devices in gynecologic procedures including endometrial ablation, gynecologic malignancies, loop electrode excision procedure (LEEP) for example in CIN treatment, using laparoscopy in infertility, hysteroscopic procedures using monopolar and bipolar resectoscopes, and laparoscopic ovarian drilling (LOD) for pcos. Categorization of various electrosurgical devices could lead to less complications and optimize evidence-based gynecological practice. 
In this study, we reviewed the basic concepts of electrosurgery and review the recent evidence on clinical application of electrosurgical devices currently used in endometrial ablation and loop electrode excision procedure (LEEP).

\section{Basic Physical Principles and Concepts}

Electrosurgery differs from electrocautery, which is based on using a heated instrument to reach the desired clinical effect. In Electrosurgery, the delivered current heats the tissue as it passes through. To better understand the biophysics of Electrosurgery, one should learn the basic underlying physical principles governing electrical circuits and energy. Electrosurgery is based on Ohm's law that describes the direct proportional relationship of the current to the potential difference between 2 points and the constant of proportionality as the resistance. All biological tissues have inherent resistance to electrical currents. The electrosurgical equipment generates the required voltage and the current is delivered via one electrode tip and returned via a specific electrode to the generator. Electrosurgical units (ESUs) use alternating current and increase the input frequencies of around $50 \mathrm{~Hz}$ to more than $500000 \mathrm{~Hz}$ to decrease the chance of probable muscle and nerve stimulation which could occur at frequencies below $100000 \mathrm{~Hz}$ and is known as Faradic effect. When the electrical current meets resistance, heat is generated within tissues according to Joules law as a function of electrical power and time multiplied, and this leads to cutting, fulguration, or desiccation depending on the duration, tissue conductivity, electrode surface area, and proximity (3).

A new research revealed that using a novel electrosurgical unit with nanostructured-doped diamondlike carbon (DLC-Cu) thin films for ablating tumors, can decrease excessive thermal injury and tissue adherence in the liver (4).

Newer electrosurgical units can modify the generated voltage based on the variable tissue resistance to maintain the desired power and heat produced at the site.

Power output can be set into cut, coagulate, and blend waveforms in most modern electrosurgical generators. Cut usually provides an almost low-voltage continuous sine wave output, whereas coagulation mode generates highvoltage interrupted outputs that could be used to reach desired clinical effect. In addition, surgeons could produce a modified cut current by setting the generator at the blend mode as needed.

From the Monopolar Electrosurgical Devices to Newer Modification Designs

Electrosurgical units (ESUs) could be categorized into monopolar and bipolar based on their circuit design and return electrode. There are 2 electrodes in monopolar systems, one as the active and the other as a dispersive pad or patient plate, which must be located far from the surgical site and is relatively large in size. Various clinical outcomes could result depending on the shape of the active electrode and surface area in contact with the tissues. Monopolar electrodes come in various shapes and forms; those with narrow or bladed tips are usually designed for cutting, and those with larger surface in forms of grasper or ball tips produce outputs suitable for desiccation and hemostasis. An insufficient contact with patient plate could result in high current density and severe burns, so proper use of gels to enhance conductivity and a reliable electrode monitoring systems are of vital importance. To lower the complication rates relating to monopolar electrosurgical systems and to improve accuracy, several innovations have been developed over the past 2 decades. In this part, we briefly review the main innovations including bipolar electrodes and vessel sealing systems.

To lower the rates of complications relating to the dispersive electrode site burn and to improve accuracy, in bipolar electrosurgical systems both electrodes are located in the same surgical device, and the only part of patient's body involved in the electrical circuit is the target tissue between the 2 electrodes. Bipolar systems have offered reliable and safe outcomes in coagulation and desiccation procedures with reduced iatrogenic complications due to unintended heat spread; yet, there remains a technical challenge about the cutting function of these devices. Innovations to address this challenge are in the evolving technological domain with introduction of new instrument tips and multifunctional devices, particularly in minimally invasive procedures.

Recently, bipolar and ultrasonic hemostatic vessel sealing devices with simultaneous cutting capabilities have revolutionized laparoscopic procedures. ENSEAL ${ }^{\circledR}$ G2 Tissue Sealers as the new generation of advanced bipolar devices, patented by ETHICON (Ethicon Endo-Surgery Inc., Cincinnati, $\mathrm{OH}$ ), have been designed to significantly improve cutting precision, reduce thermal injury using temperature-regulating characteristics of the jaws, and finally present a more cost-effective alternative in the field of minimally invasive surgery (5). However, comparative studies on the superiority of advanced bipolar devices, compared to one another, have failed to reach a general consensus (6). A recent randomized clinical trial observed similar primary and secondary outcomes in patients undergoing total laparoscopic hysterectomy using LigaSure, HALO PKS, and ENSEAL, respectively. (7)

On the other hand, various reports exist on specific advantages and disadvantages of certain aspects of these technologies. Newcomb et al. compared electrosurgical and ultrasonic vessel sealing devices and reported minimal seal failures using LigaSure V with LigaSure Vessel Sealing Generator, LigaSure V with Force Triad Generator, EnSeal Tissue Sealing, and Hemostasis System. These devices also produced the highest burst pressure and LigaSure V, with Force Triad Generator having the shortest mean seal time for large and medium sized vessels (8). In another study on advanced bipolar devices, ENSEAL G2 sealers have been reported to produce stronger and more consistent sealing with more uniform compression compared to LigaSure (9). Moreover, Seehofer et al. have reported the use of integrated bipolar and ultrasonic scissors with potential better dissection speeds and similar sealing efficacy of conventional bipolar clamps (10). Compressive force monitoring has also been introduced as a technological 
modification in some bipolar laparoscopic devices to ensure adequate and optimal burst pressure and improve sealing quality (11).

Monopolar and bipolar resectoscopes have also been introduced in gynecologic and urologic procedures mainly to ablate tissues and extract biopsies. Similar reproductive outcomes have recently been observed in hysteroscopic procedures using monopolar and bipolar resectoscopes. However, risk of hyponatremia has been found to be less in procedures performed by bipolar instruments (12).

Complications of Electrosurgery and Basic Safety Measures

During the past several decades, a variety of engineering innovations has been introduced to address the common complications that occurred in electrosurgical procedures. Ground point, alternate and dispersive electrode burns were the most common complications before 1970. During that period, a common complication of electrosurgery was ground site burns caused by ground-referenced generators and potential contact with a pass to ground, which has been modified by development of ground isolated systems (13). Moreover, to prevent electrical burns at the site of the dispersive pads, return electrode monitoring system could be used to deactivate the device in case the contact between the dispersive pad and patient's body is interrupted.

More recently, unwanted electrothermal injury due to direct coupling and insulation failure has become the topic of ongoing investigation. The repetitive use of instruments could lead to defects in insulating coating that might lead to serious complications, particularly in laparoscopy. (14) Careful maintenance of the electrosurgical devices and use of disposable instruments could dramatically reduce accidental burns caused by insulation failure. Furthermore, active electrode monitoring could detect stray energy and shut down the generator in case of capacitive coupling and insulation failure, which might be invisible to the naked eyes of experienced surgeons; therefore, preventing deleterious outcomes due to high concentration of currents was unintentionally done through small defects (15). In addition, unintended direct application may occur during electrosurgical procedures, and the surgical team must pay careful attention to surrounding tissues and potential conductive instruments in proximity before activation of the electrodes.

Most important basic safety measures to prevent complications, while using electrosurgical devices are as follows: using the manufactures recommended cables; careful inspection of the device; looking for insulation defects; using the lowest voltage, power setting and application time to achieve the desired effects; alternating between desiccation and incision; using a monitored return electrode or active electrode monitoring systems; not using hybrid metal-plastic systems; placing the electrodes in their safety holster and not activating the system, while the electrodes are not in contact with tissues to avoid open circuitry (14).

Evidence suggests that electrosurgical techniques could be safely used during pregnancy. Frequency of the produced currents could not lead to contractions, and fetus is protected by amniotic fluid. However, great caution should be taken to avoid direct contact of electrodes with the fetal tissues during any procedure as it might lead to thermal injuries(16).

\section{Hyperthermic Fibroid and Endometrial Ablation}

Since 1980s, endometrial ablation has been introduced in treatment of menorrhagia in patients who do not respond to standard medical therapies. Over the years, use of radiofrequency ablation (RFA) and coagulative necrosis with RF energy has been studied as a therapeutic option in managing uterine fibroids $(17,18)$. In the late 1980s, a technique referred to as myoma coagulation was introduced to ablate fibroids using neodynium: yttrium aluminium garnet (Nd:YAG) laser as the source of hyperthermic energy. Later, bipolar radiofrequency needle electrodes were used as an alternative to laser. Despite the significant devascularization and decrease in size of the fibroids, these techniques did not gain clinical acceptance due to risk of developing serosal injury, myometrial weakening, and dense fibrous adhesions (19).

In practice, endometrial ablation with bipolar radiofrequency is preferable in the treatment of heavy menstrual bleeding compared to balloon of the endometrium with respect to amenorrhea, patient satisfaction, and patient quality of life (20).

During the last 10 years, balloon and microwave thermal ablation have been introduced with less invasiveness, more safety, and shorter hospital stays (21). There is an evergrowing body of evidence indicating the efficacy of hyperthermic fibroid ablation and improved quality of life among patients undergoing such procedure(22). Costeffectiveness studies on thermal balloon endometrial ablation (TBEA) and microwave endometrial ablation (MEA) highlight the superiority of these second generation techniques (23).

Safe and successful outpatient TBEA under local anesthesia with high patient satisfaction has been reported (24). Menotreat and thermablate have been studied as wellaccepted and safe systems in the treatment of dysfunctional uterine bleeding $(25,26)$. Four thermal endometrial balloon ablation systems are currently used, yet in developing countries, most of these technologies are not easily available to the public. However, effectiveness and accessibility of ThermaChoice and Cavaterm have been tested in India and Iran, with satisfactory results $(27,28)$. In a recent randomized controlled trial, no significant difference was observed in quality of life, hysterectomy rates, and patient satisfaction 5 years after treatment with MEA $^{\mathrm{TM}}$ compared to Thermachoice 3. However, they reported higher costs and slower treatment times in patients undergoing TBEA with compared to those treated with microwave endometrial ablation (29).

\section{Electrosurgery in Laparoscopic Procedures in Endometrial and Cervical Cancer}

Conventional and robot-assisted laparoscopic procedures have been used as acceptable alternatives to laparotomy as the traditional surgical approach to endometrial cancer treatment and staging (30). These approaches have proved particularly promising in early stages of endometrial 
cancer; and review of the literature highlights fewer complications, less blood loss, and shorter stay in hospital in those patients undergoing laparoscopic procedures. There is a lack of conclusive evidence on the survival rates,particularly in more advanced stages; however, no significant difference has been reported by the few available studies $(31,32)$. Moreover, the optimal surgical approach regarding the removal of lymph nodes for staging of endometrial cancer is a matter of ongoing debate in gynecologic literature (33). Laparoscopic approach and sentinel lymph node mapping has been suggested as an accurate method in early stages of cervical and endometrial cancers $(34,35)$.

The field of minimally invasive surgery and devices used keep on improving and evolving. There have been several advancements in electrosurgical devices used in laparoscopic procedures to manage endometrial cancer. The conventional monopolar and bipolar devices could cause thermal damage, so innovations have been made to address this issue. One of the most active surgical teams in performing laparoscopic radical hysterectomy has developed a pulsed bipolar system. They have reported better surgical outcomes including less complications, operation time, and blood loss. In line with these findings, Lee et al. indicated that pulsed bipolar systems could offer advantages in the management of early cervical carcinoma in patients undergoing laparoscopic radical hysterectomy and pelvic lymphadenectomy (36). Laparasonic devices including harmonic scalpel and coagulating shears have also been compared to electrosurgical devices in lymph node dissection and laparoscopic hysterectomy. Holub et al. reported these devices as more cost-effective alternatives with less thermal injury in lymph node dissection procedures (37). A randomized trial reported the superiority of a multifunctional instrument that integrates ultrasonic waves and advanced bipolar energy with simultaneous sealing and dissection capabilities. They found that these modified electrosurgical devices could save time and result in less postoperative pain to treat early stages of cervical cancer in patients undergoing laparoscopic radical hysterectomy and lymphadenectomy(38).

\section{Electrosurgical Principles of Loop Electrode Excision Procedure (LEEP)}

Use of electrosurgical wire loop biopsy, also referred to as large loop excision of the transformation zone (LLETZ), is established as an outpatient gynecological procedure. It helps gain adequate biopsy specimen from abnormal cervical tissue and canal that might not be fully visible during colposcopy. It is extensively used in definite diagnosis and treatment of moderate to severe cervical dysplasia (CIN II/III).

There is a lack of evidence the comparison of different electrosurgical generators, long-term outcomes, and rates of complication of each device, thus, basic electrosurgical safety measures should be taken to reduce probable adverse outcomes.

Prior excisional cervical procedures have been reported as a risk factor for preterm delivery in the literature. Despite the significant association observed with subsequent preterm deliveries, low birth weight, and preterm premature rupture of the membranes, increasing LEEP depth or volume have not been associated with increased preterm birth rate, as reported by a meta-analysis published in 2014 (39). In another recent systematic review of the literature, Conner et al. assessed whether the increased risk of preterm birth is associated with risk factors related to cervical dysplasia rather than loop electrosurgical excision procedure itself. They concluded that LEEP itself might not be an independent risk factor, and common risk factors for preterm birth and dysplasia could account for the observed association (40). However, this finding remains to be fully investigated by further carefully designed and sufficiently powered studies.

\section{Electrosurgical Techniques and Treatment of Infertility}

Electrosurgical approaches have been also used in the management of polycystic ovary syndrome (PCOS) as the most common cause of anovulatory infertility (41). In PCOS cases unresponsive to clomiphene, laparoscopic ovarian drilling (LOD) has been recommended as a safe and cost-effective alternative to other treatment modalities including the use of gonadotropins. Using monopolar electrosurgical devices remains the most common method in LOD procedures; however, using bipolar devices have been reported with comparable clinical outcomes (42). However, recent evidence suggests that combination of oral therapies such as clomiphene plus metformin or aromatase inhibitors, could represent a more conservative and successful alternative to LOD as the second line treatment in these women (43).

Electrocoagulation and electroexcision of endometriosis is another example of electrosurgery in patients suffering infertility. There remains great challenges in precise electrocautery near vital structures affected by endometriosis and these techniques could usually only be safely used in managing superficial peritoneal disease. Some authors have indicated fewer relapses when using bipolar electrocoagulation compared to excision (44). On the other hand, excision has been described as more effective in reducing pelvic pain; moreover, low complication rate and higher quality of life have also been reported in a cohort of women undergoing laparoscopic excision of endometriosis (45). Nonetheless, further randomized prospective studies are required to fully establish the value of these surgical techniques and their indications according to patients' criteria (46).

\section{Conclusion}

In this paper, we provided a review of basic concepts of electrosurgery and important clinical applications of electrosurgical devices in gynecologic procedures. Considering the extensive use of these technologies in reproductive related surgeries, gynecologists must learn the basics in more detail and update their knowledge on the growing body of evidence regarding the advancements of these technologies to reduce potential complications and select the most cost-effective treatment options for each patient. Some aspects, particularly comparison of advanced 
electrosurgical technologies, remain to be fully elucidated, and further well-designed trials are warranted to address the current gaps in the literature.

\section{Acknowledgments}

We would like to thank Dr. Alireza Kazemi for all his conceptual support.

\section{Conflict of Interests}

The authors declare that they have no competing interests.

\section{References}

1. Taheri A, Mansoori P, Sandoval LF, Feldman SR, Pearce D, Williford PM. Electrosurgery: part I. Basics and principles. J Am Acad Dermato. 2014;70(4):591.e1-14; quiz 605-6.

2. Al-Benna S. Albucasis, a tenth-century scholar, physician and surgeon: His role in the history of plastic and reconstructive surgery. Eur J Plastic Sur. 2012;35(5):379-87.

3. Massarweh NN, Cosgriff N, Slakey DP. Electrosurgery: History, Principles, and Current and Future Uses. J Am College Surg. 2006;202(3):520-30.

4. Shen YD, Lin LH, Chiang HJ, Ou KL, Cheng HY. Research of electrosurgical unit with novel antiadhesion composite thin film for tumor ablation: Microstructural characteristics, thermal conduction properties, and biological behaviors. J Biomed Mater Res Part B Appl. Biomater. 2016;104(1):96-105.

5. Kondrup JD, Anderson F, Quick B. Use of the ENSEAL(R) G2 tissue sealer in gynecologic surgery. Surg Tech Int. 2013;23:191-5.

6. Lyons SD, Law KS. Laparoscopic vessel sealing technologies. J Minim Invasive Gynecol. 2013;20(3):301-7.

7. Aytan H, Nazik H, Narin R, Api M, Tok EC. Comparison of the use of LigaSure, HALO PKS cutting forceps, and ENSEAL tissue sealer in total laparoscopic hysterectomy: a randomized trial. Jmig. 2014;21(4):650-5.

8. Newcomb WL, Hope WW, Schmelzer TM, Heath JJ, Norton HJ, Lincourt $\mathrm{AE}$, et al. Comparison of blood vessel sealing among new electrosurgical and ultrasonic devices. Surg.Endosc. 2009;23(1):90-6.

9. Chekan EG, Davison MA, Singleton DW, Mennone JZ, Hinoul P. Consistency and sealing of advanced bipolar tissue sealers. Med Devices (Auckl). 2015;8:193-9.

10. Seehofer D, Mogl M, Boas-Knoop S, Unger J, Schirmeier A, Chopra $\mathrm{S}$, et al. Safety and efficacy of new integrated bipolar and ultrasonic scissors compared to conventional laparoscopic $5-\mathrm{mm}$ sealing and cutting instruments. Surg.Endosc. 2012;26(9):2541-9.

11. Chen RK, Chastagner MW, Geiger JD, Shih AJ. Bipolar electrosurgical vessel-sealing device with compressive force monitoring. J Biomech eng. 2014;136(6):061001.

12. Roy KK, Kansal Y, Subbaiah M, Kumar S, Sharma JB, Singh N. Hysteroscopic septal resection using unipolar resectoscope versus bipolar resectoscope: Prospective, randomized study. J Obstet Gynecol Re. 2015;41(6):952-6.

13. Sankaranarayanan G, Resapu RR, Jones DB, Schwaitzberg S, De S. Common uses and cited complications of energy in surgery. Surg.Endosc. 2013;27(9):3056-72.

14. Alkatout I, Schollmeyer T, Hawaldar NA, Sharma N, Mettler L. Principles and safety measures of electrosurgery in laparoscopy. JSLS. 2012;16(1):130-9.

15. Vilos GA, Newton DW, Odell RC, Abu-Rafea B, Vilos AG. Characterization and mitigation of stray radiofrequency currents during monopolar resectoscopic electrosurgery. J Minim Invasive Gynecol. 2006;13(2):134-40.

16. Feldman L, Fuchshuber PR, Jones DB. The SAGES manual on the fundamental use of surgical energy (FUSE): Springer; 2012.

17. Recaldini C, Carrafiello G, Lagana D, Cuffari S, Bergamini V, Ghezzi $\mathrm{F}$, et al. Percutaneous sonographically guided radiofrequency ablation of medium-sized fibroids: feasibility study. AJR. 2007;189(6):1303-6.

18. Brucker SY, Hahn M, Kraemer D, Taran FA, Isaacson KB, Kramer B. Laparoscopic radiofrequency volumetric thermal ablation of fibroids versus laparoscopic myomectomy. Int $\mathrm{J}$ Gynaecol Obstet 2014;125(3):261-5.

19. Donnez J, Squifflet J, Polet R, Nisolle M. Laparoscopic myolysis.
Hum Reprod Update. 2000;6(6):609-13.

20. Penninx JP, Herman MC, Kruitwagen RF, Ter Haar AJ, Mol BW, Bongers MY. Bipolar versus balloon endometrial ablation in the office: a randomized controlled trial. EJOG. 2016;196:52-6.

21. Madhu CK, Nattey J, Naeem T. Second generation endometrial ablation techniques: an audit of clinical practice. Arch Gynecol Obstet. 2009;280(4):599-602.

22. Jones S, O'Donovan P, Toub D. Radiofrequency ablation for treatment of symptomatic uterine fibroids.OGIJ. 2012;2012:194839.

23. Garside R, Stein K, Wyatt K, Round A, Price A. The effectiveness and cost-effectiveness of microwave and thermal balloon endometrial ablation for heavy menstrual bleeding: a systematic review and economic modelling. HTA. 2004;8(3):1-155.

24. Varma R, Soneja H, Samuel N, Sangha E, Clark TJ, Gupta JK. Outpatient Thermachoice endometrial balloon ablation: long-term, prognostic and quality-of-life measures. Gynecol Obstet Investig. 2010;70(3):145-8.

25. Prasad P, Powell MC. Prospective observational study of Thermablate Endometrial Ablation System as an outpatient procedure. J Minim Invasive Gynecol. 2008;15(4):476-9.

26. Vihko KK, Raitala R, Taina E. Endometrial thermoablation for treatment of menorrhagia: comparison of two methods in outpatient setting. Acta Obstet Gynecol Scand. 2003;82(3):269-74.

27. Pai RD. Thermal balloon endometrial ablation in dysfunctional uterine bleeding. Surg Endosc. 2009;1(1):31-3.

28. Bouzari Z, Yazdani S, Azimi S, Delavar MA. Thermal balloon endometrial ablation in the treatment of heavy menstrual bleeding. Med Arch. 2014;68(6):411-3.

29. Sambrook A, Elders A, Cooper K. Microwave endometrial ablation versus thermal balloon endometrial ablation (MEATBall): 5-year follow up of a randomised controlled trial. BJOG. 2014;121(6):747-53.

30. Ghaemmaghami F, Aminimoghaddam S, Modares-Gilani M, Mousavi A, Khazaeipour Z, Fereidoni F. Assessment of gross examination and frozen section of uterine specimen in endometrial cancer patients. Arch Gynecol Obstet. 2010;282(6):685-9.

31. Mourits MJ, Bijen CB, Arts HJ, ter Brugge HG, van der Sijde R, Paulsen L, et al. Safety of laparoscopy versus laparotomy in early-stage endometrial cancer: a randomised trial. Lancet Oncol. 2010;11(8):76371.

32. Aminimoghaddam S, Shahrabi-Farahani M, Mohajeri-Tehrani M, Amiri P, Fereidooni F, Larijani B, et al. Epistatic interaction between adiponectin and survivin gene polymorphisms in endometrial carcinoma. Pathol Res Pract. 2015;211(4):293-7.

33. Khoury-Collado F, Clair CS, Abu-Rustum NR. Sentinel Lymph Node Mapping in Endometrial Cancer: An Update. The Oncologist. 2016: 0473.

34. Allameh T, Hashemi V, Mohammadizadeh F, Behnamfar F. Sentinel lymph node mapping in early stage of endometrial and cervical cancers. JRMS. 2015;20(2):169.

35. Aminimoghaddam S, Shadman L, Hashemi RS, Mohammadi Z, Mahmoudzadeh F. Sentinel Lymph Node Detection Using Methylene Blue in Iranian Patients with Early Stage Cervical Cancer. J Appl Sci. 2015;15(3):582.

36. Lee CL, Huang KG, Wang CJ, Lee PS, Hwang LL. Laparoscopic radical hysterectomy using pulsed bipolar system: comparison with conventional bipolar electrosurgery. Gynecol Oncol. 2007;105(3):6204.

37. Holub Z, Jabor A, Kliment L, Voracek J, Lukac J, Barany B. Laparoscopic staging of endometrial cancer using laparosonic instruments: comparison with electrosurgery. Eur J Obstet Gynecol Reprod Biol. 2001;100(1):81-6.

38. Fagotti A, Vizzielli G, Fanfani F, Gallotta V, Rossitto C, Costantini B, et al. Randomized study comparing use of Thunderbeat technology vs standard electrosurgery during laparoscopic radical hysterectomy and pelvic lymphadenectomy for gynecologic cancer. J Minim Invasive Gynecol. 2014;21(3):447-53.

39. Jin G, LanLan Z, Li C, Dan Z. Pregnancy outcome following loop electrosurgical excision procedure (LEEP) a systematic review and meta-analysis. Arch Gynecol Obstet. 2014;289(1):85-99.

40. Conner SN, Frey HA, Cahill AG, Macones GA, Colditz GA, Tuuli MG. Loop electrosurgical excision procedure and risk of preterm birth a systematic review and meta-analysis. Obstet Gynecol. 2014;123(4):752-61.

41. Tehrani MM, Parvizi M, Moghadam SA, Heshmat R, Shaban NejadKhas Z, Golchin M. The prevalence of polycystic ovary syndrome in 
Iranian women with gestational diabetes: a pilot study. Journal of Diabetes \& Metabolic Disorders. 2009. p57-63.

42. Mitra S, Nayak PK, Agrawal S. Laparoscopic ovarian drilling: an alternative but not the ultimate in the management of polycystic ovary syndrome. J Nat Sci Biol Med. 2015;6(1):40.

43. Hashim HA, Al-Inany H, De Vos M, Tournaye H. Three decades after Gjönnaess's laparoscopic ovarian drilling for treatment of PCOS; what do we know? An evidence-based approach. Arch Gynecol Obstet. 2013;288(2):409-22.

44. Radosa MP, Bernardi TS, Georgiev I, Diebolder H, Camara O, Runnebaum IB. Coagulation versus excision of primary superficial endometriosis: a 2-year follow-up. Eur J Obstet Gynecol Reprod Biol. 2010;150(2):195-8.

45. Roman JD. Surgical treatment of endometriosis in private practice: cohort study with mean follow-up of 3 years.Jmig. 2010;17(1):42-6.

46. Shahrabi-Farahani M, Shahbazi S, Mahdian R, Amini-Moghaddam S. $\mathrm{K}-\mathrm{Ras} 4 \mathrm{~A}$ Transcript variant is up-regulated in eutopic endometrium of endometriosis patients during proliferative phase of menstrual cycle. Arch Gynecol Obstet. 2015;292(1):225-9. 